MATEC Web of Conferences 22,01055 (2015)

DOI: $10.1051 /$ matec conf/ 20152201055

(C) Owned by the authors, published by EDP Sciences, 2015

\title{
Applications of ZigBee Technology in the Safety Monitoring System of Low Gas Pipeline Transportation
}

\author{
Deyu Wei \& Hucheng Wu \\ Henan College of Industry \& Information Technology, Jiaozuo, Henan, China
}

Xinhe $\mathrm{Yu}$

Henan Zhong Mei Machinery Co., Ltd., Zhengzhou, Henan, China

\begin{abstract}
The existing safety monitoring system of low gas pipeline transportation establishes a wired communication network monitoring system mainly on the basis of industrial bus. It has problems such as large transmission signal attenuation, complex wiring, high-labor intensity, inconvenient installation and maintenance, high maintenance cost, and so on. Featuring low cost, power-saving, reliability, stability and flexibility, the wireless sensor network established by ZigBee wireless communication technology can realize the real-time all-dimensional dynamic monitoring on parameters of low gas pipeline transportation system and overcome the shortcomings and deficiencies of wired network system.
\end{abstract}

Keywords: low gas pipeline transportation; sensor; ZigBee wireless network; ZigBee protocol stack

\section{INTRODUCTION}

The design of the safety guarantee system of low concentration coal gas pipeline transportation should follow the principle of "fire and explosion venting, explosion suppression and resistance, multilevel protection and safety insurance". The safety guarantee system of low concentration coal gas pipeline transportation of wired network has been developed in recent years. But the wired network system also has certain problems. When the monitoring center is distant from monitor points, the transmission signal attenuation is relatively strong with complex wiring, high-labor intensity, inconvenient installation and maintenance, and high maintenance cost.

ZigBee technology is mainly used to establish a wireless personal area network of low bit rate. Members of the network are called the node equipment. The acquisition of all kinds of information in monitoring areas can be realized by setting a large number of sensor nodes. The main advantages include extremely low cost of system, safe data transmission, flexible networking and large network capacity.

\section{PRINCIPLE OF THE GROUND GAS EMPTYING SYSTEM}

As shown in Figure 1, sensors of the front end of gas drainage include a flame sensor, a pressure sensor and a water-level sensor. The rear end also has a flame sensor, a pressure sensor, and a water-level sensor as well as protective devices such as an automatic powder-spray device for explosion suppression,

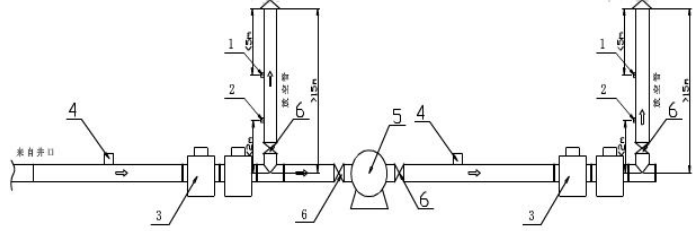

1-flame sensor; 2-pressure sensor; 3-water blocking device of fire and explosion venting; 4-automatic powder-spray device for explosion suppression; 5-vacuum pump; 6-stop valve

Figure 1. Installation diagram of the safety device of ground gas emptying system.

vacuum pump and stop valve.

\section{SYSTEM STRUCTURE}

The system structure is shown in Figure 2. In this system, two ZigBee terminals (RFD) and a ZigBee coordinator (FFD) compose a star-shaped WSN Parameters (flame, pressure, level) are collected by sensors on terminal nodes, and wirelessly transmitted from the terminals to coordinators, and then they are transmitted to the supervisory control computer through a USB interface.

The ZigBee terminal node (RFD) consists of four parts, namely data acquisition sensor, processing module, wireless communication module and power module. The ZigBee coordinator (FFD) is composed of USB communication module, processing module, wireless communication module and alarm module. 


\section{MATEC Web of Conferences}
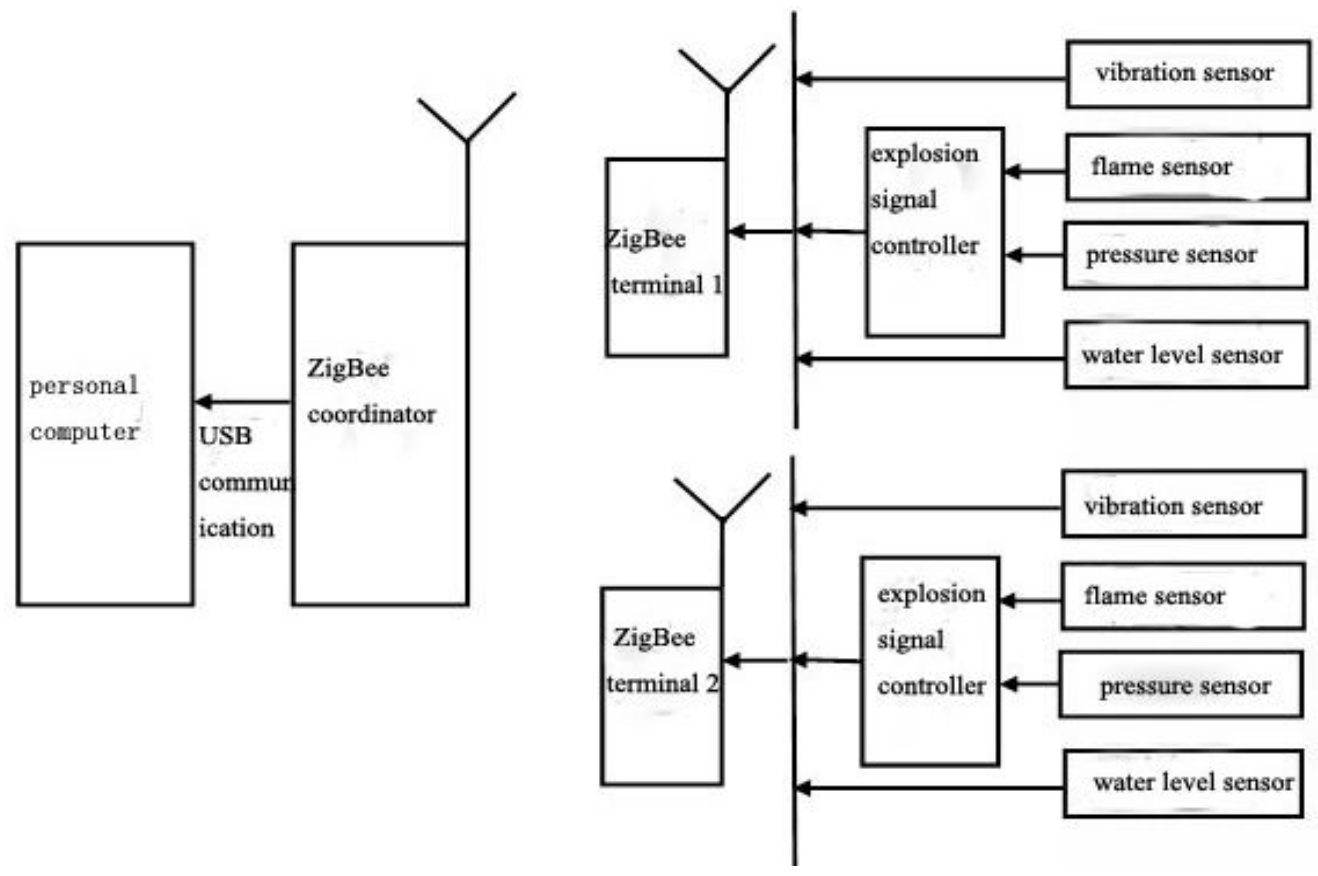

Figure 2. System structures

\section{HARDWARE DESIGN OF TERMINAL NODES}

\subsection{Selection of sensors}

Flame and pressure sensors adopt the explosion signal controller of intrinsic safety type. As shown in Figure 3 , two flame sensors are connected to the switch input channel of the controller and the other two sensors are connected to the analog input channel of the controller. Signals of four channels are outputted, which are respectively explosion signals, status signals, control signals and pressure signals. The reaction of explosion signal output is used to judge whether there are explosions. If there are explosions, voltage of $0 \mathrm{~V}$ is outputted. If there is no explosions, voltage of $4 \mathrm{~V}$ is outputted. The reaction of status signals is the working condition of the controller. It can output $4 \mathrm{~V}$ in normal working condition or output $0 \mathrm{~V}$ if the controller breaks down. Control signals are used to control the powder-spray of the automatic explosion suppression device. It can output $4 \mathrm{~V}$ when there are explosions or output $0 \mathrm{~V}$ when there are no explosions. The controller of pressure signals includes pressure signals of two channels. It can output the monitored pressure value of the first channel when there are no explosions, and it can output the maximum pressure of the two channels when there are explosions. Frequency signals are outputted as well.

In order to ensure the reliability of monitoring signals, the mechanical shock-wave sensors are built up to transmit shock-wave signals in pipeline to the explosion signal controller in time. When burning or explosion signals are monitored, ignition signals of the

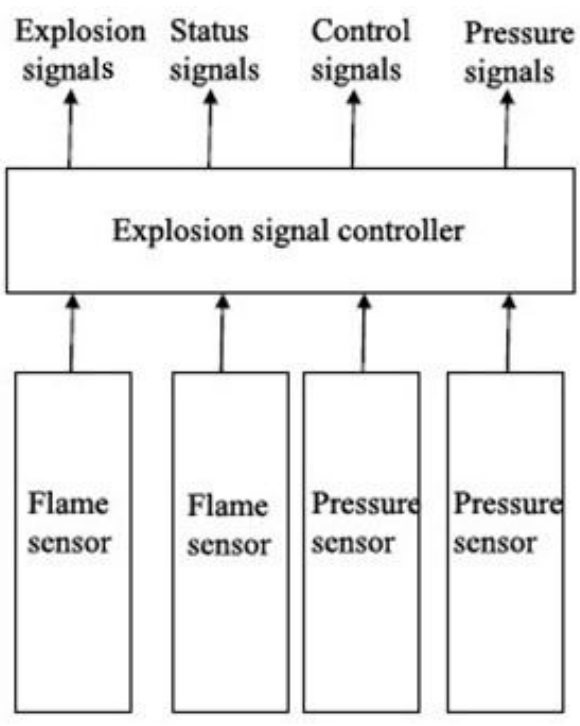

Figure 3. Generator of explosion signals

automatic explosion resistance device are emitted from the explosion signal controller and then immediately cut off by the shut-down valve.

The water-level sensor is the GUY1.2 liquid level sensor with the operating voltage of DC12 24 V and the measurement range of $0 \sim 1.2 \mathrm{~m}$. Frequency signals are outputted with the operating voltage of DC $0 \sim 5 \mathrm{v}$. The intrinsic safety type of mining is exhibited. 
ICETA 2015

\subsection{ZigBee chip selection}

CC2530 is a new generation of SoC chip based on ZigBee/IEE802.15.4 standard developed by IT Company. It is integrated with a high-performance RF transceiver, an optimized low-consumption 8051 microprocessor which has an $8 \mathrm{kB}$ RAM and $256 \mathrm{kB}$ flash memory, including 7-12 bits ADC of 8 channels, 2 USART and 21 general I/O interfaces.

CC2530 is a wireless transmitting chip that has the function of being able to accomplish all functions required for this design without the need of additional control chips. So, CC2530 is adopted to realize the sending and receiving of wireless signals.

CC2530 has 8 alternative AD channels that are used to connect to 8 kinds of sensors. It can measure a variety of different physical quantities. This design only includes the measurement of 6 kinds of physical quantities, namely 4 signals outputted by the explosion signal controller, the water-level sensor and the mechanical shock wave sensor.

\subsection{Hardware design of ZigBee wireless module}

Figure 4 is the hardware system diagram of ZigBee wireless module. Two crystal oscillators are permanently connected with the CC2530 chip. The one working frequency is $32 \mathrm{MHz}$ and another is $32.786 \mathrm{MHz}$. The $32 \mathrm{MHz}$ crystal oscillator is the main frequency of the system, which provides clock pulses for the normal operation of the control system. The $32.786 \mathrm{MHz}$ crystal oscillator provides clock pulses for the sleep timer and the watchdog timer. A power filter is also added to the circuit so as to improve the quality of power supply and provide $\mathrm{AD}$ conversion with more accurately external reference voltage. The port $\mathrm{P} 0-0-\mathrm{P} 0-7$ is adopted as the AD sampling port, which sends $A D$ sampling data to the coordinator through wireless network. The coordinator only receives data and transmits data to the data processing host through serial ports. Therefore, IO ports $\mathrm{P} 0-2$ and $\mathrm{P} 0-3$ are used as serial interfaces.

\section{SYSTEM SOFTWARE DESIGN}

This system adopts the star-shaped networking scheme. A ZigBee gateway and multiple ZigBee data nodes are adopted in the software design of the system. The software includes three parts, namely acquisition and transmission of ZigBee data nodes, the ZigBee gateway, and the upper computer.

AIR7.60 and AIR7.60 adopted by the development environment of the design are softwares of the application layer compiled on the basis of Z-STACK-2.4.0-1.4.0 protocol stack. The system is composed of a star-shaped network consisting six nodes: one coordinator which is in charge of network establishment, three routers and two terminal component networks.

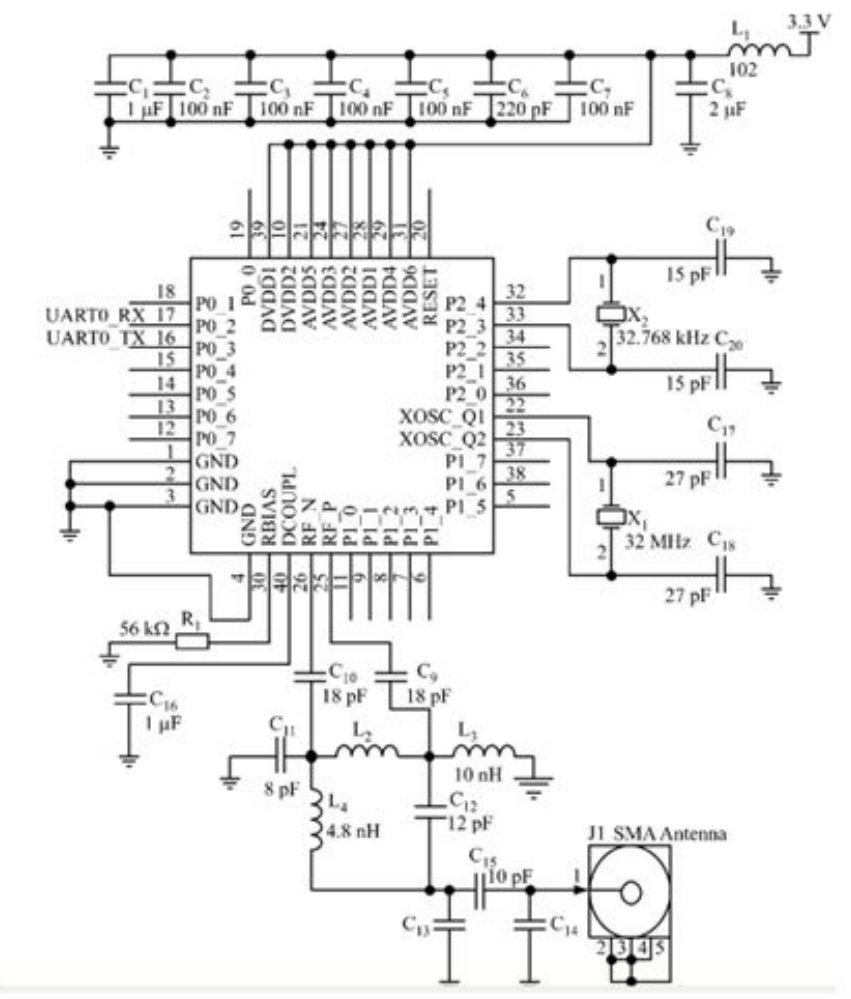

Figure 4. Hardware system of ZigBee wireless module 


\section{MATEC Web of Conferences}

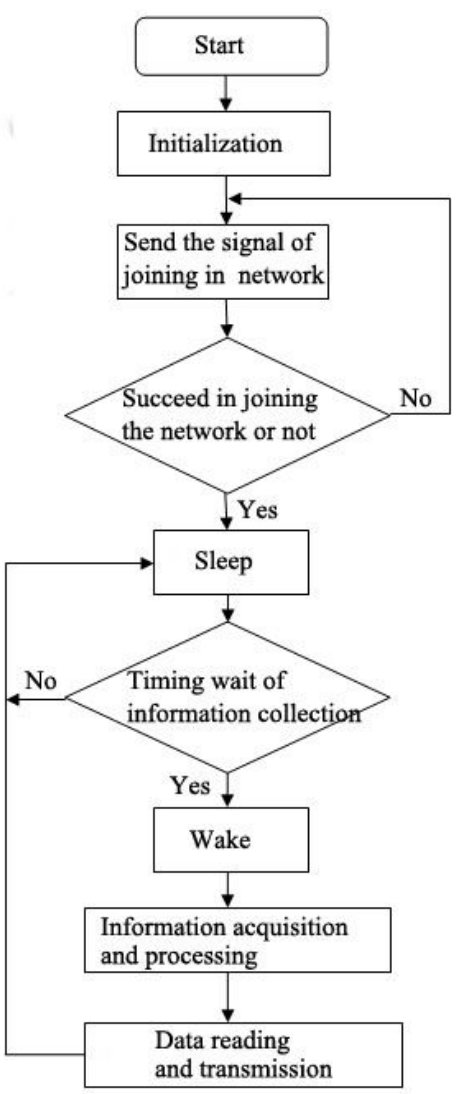

Figure 5. Program chart of the transmitting terminal.

\subsection{Acquisition and transmission of ZigBee data}

Data of measuring flame, pressure, water-level and mechanical shock-wave sensors can be collected with the CC2530 module. Analog signals and switch signals outputted by sensors are converted into digital signals through the signal-conditioning circuit. CC2530 emits data after a processing through the CC2530 transmitting antenna.

In order to realize the wireless transmission, a program is integrated in CC2530 chips of the transmitting terminal and the receiving terminal. As shown in Figure 5, in the CC2530 chip of the transmitting terminal, the chip will build up a network after the initialization of each module in the program. If there are sensors requesting to join the network, the next step will be carried on. Otherwise, the detection will be carried on. The chip will then store node addresses of sensors. And the data can be transmitted to the receiving terminal by the chip through key inputting at this moment. The program chart of the transmitting terminal is shown in Figure 5.

In the CC2530 chip of the receiving terminal, the chip will first search the network and then try to join the network after the initialization of each module in

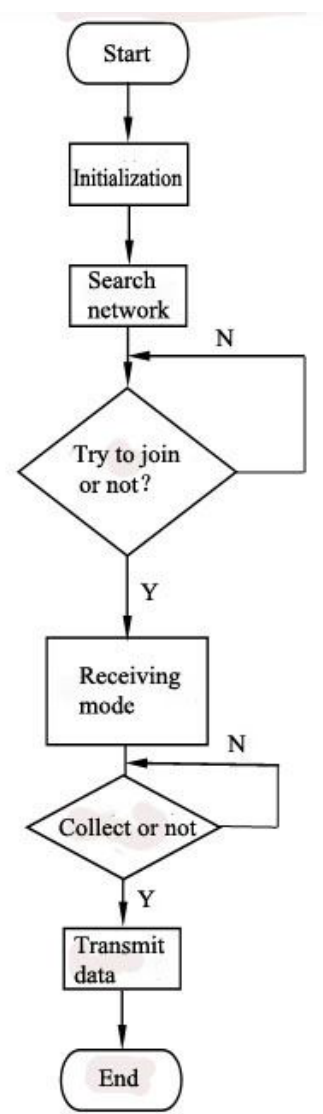

Figure 6. Program chart of the receiving terminal.

the program. If the chip successfully joins the network, the chip will be in the receiving mode. Otherwise, it will continue to join the network. In the receiving mode, the system will determine whether data should be collected. If the chip collects data, it will transmit data to functional modules. Otherwise, the detection will be carried on. The program chart of the receiving terminal is shown in Figure 6.

\subsection{Program design of network coordinator}

Coordinator nodes are used to manage the entire network. It not only receives commands from the computer terminal and transmits to other nodes, but also receives information transmitted from other nodes and uploads it to computer for display through RS232 serial ports.

The main function is presented below:

While TRUE

If RfRxFlag $==1$

RfRxFlag $\leftarrow 0$

If macRx.sourceShortAddr $<201$

Extract data () //extract data

printFlag $\leftarrow$ TO_LCD12864; // set the printf output function as LCD output mode

Print data () // output received data on LCD screen 
printFlag $\leftarrow$ TO_UART; // set the printf output function as serial port transmitting mode

Send data () // transmit received data to serial ports

End if

LED $\leftarrow$ LED

End if

End while

\section{CONCLUSION}

Featuring low cost, low complexity, low power dissipation and Ad-Hoc network, and the safety monitoring system of low gas pipeline transmission based on ZigBee, this system has obvious advantages compared to the wired system with perfect values of promotion and prospects of application.

\section{REFERENCES}

[1] Zhang, D., Li, C.L. \& Xu, Y. et al. 2010. Design of coal mine safety monitoring system based on ZigBee technology, Safety in Coal Mines, 41(7): 77-80.

[2] Yang, J. 2011. A study on the safety monitoring system of low gas pipeline transmission, Automation \& Instrumentation, 155(3): 28-31.

[3] Peng, F., Wang, J.Q. \& Shi, C.H. 2013. Design and implementation of refrigeration storage monitoring system based on ZigBee, Journal of Chinese Agricultural Mechanization, 34(9): 248-250.

[4] Li, X.Z., Hu, Z.X. \& Feng, X.H., et al. 2014. Design of pressure monitoring system of coal mine tunnel based on ZigBee, Industry and Mine Automation, 40(4): 19-22.

[5] Zhu, Y.K., Li, Z.B. \& Jiang, Y.Y., et al. 2014. Power leakage protection system of underground power grid based on ZigBee wireless sensor network, Industry and Mine Automation, 40(5): 15-17.

[6] Bo, Y.Q., Ouyang, M.S. \& Li, Y.L., et al. 2014. Hydrological information monitoring system of mines based on ZigBee, Industry and Mine Automation, 40(10): 84-87. 